\title{
Stability of Eshelby Dislocations in FCC Crystalline Nanowires
}

\author{
Seunghwa Ryu ${ }^{1 *}$ and Wei Cai ${ }^{2 *}$ \\ ${ }^{1}$ Department of Mechanical Engineering, \\ Korea Advanced Institute of Science and Technology, Daejeon 34141, Korea \\ ${ }^{2}$ Department of Mechanical Engineering, Stanford University, Stanford, CA94305, USA
}

The thermally activated escape of Eshelby dislocations in face-centered-crystal (FCC) nanowires of finite length with surface steps at the ends is investigated by combining atomistic and continuum models. The energy barriers for the dislocation escape from facecentered-cubic $\langle 110\rangle$ nanowires are predicted from atomistic models as a function of nanowire radius, escape location, and surface step orientation. The dissociation of a dislocation into partials has a significant effect on the energy barrier. The dislocation prefers to escape from the end of nanowire with an extended node, where the dissociation width is greater than the equilibrium width in the bulk. The energy barrier is further lowered if the surface step aligns with the dislocation's slip plane. A continuum line tension model that accounts for partial dislocations is constructed and benchmarked against atomistic predictions for FCC nanowires. The continuum model is then used to make predictions on the stability of Eshelby dislocations over a wide range of nanowire radii.

Keywords: Eshelby dislocation, energy barrier, atomistic simulation, line tension model

\footnotetext{
* To whom correspondence should be addressed: ryush@kaist.ac.kr.edu

*To whom correspondence should be addressed: caiwei@ stanford.edu
} 


\section{Introduction}

Owing to the large surface-to-volume ratio and directional transport properties, crystalline nanowires (NWs) have been explored for a wide range of applications including nano-electronics, nano-photonics, renewable energy, and chemical sensing (Mai et al., 2014; Palacios, 2012; Penner, 2012; Xiong et al., 2013). A fundamental understanding of their growth and deformation mechanisms is crucial for achieving a rational design of nano-devices using NWs as building blocks. NWs are often found to grow via a screw-dislocation-driven (SDD) process, resulting in a screw dislocation along the NW axis, as observed in $\mathrm{PbSe}, \mathrm{PbS}$ and Cu NWs (Bierman et al., 2008; Meng, 2012; Zhu et al., 2008). The screw dislocation provides self-perpetuating steps on the NW end surface, promoting directional growth along the NW axis. Eshelby was the first to show that the axial screw dislocation produces a twist along the NW (Eshelby, 1953; Eshelby and Stroh, 1951), which is now called the Eshelby twist, and was confirmed recently in branched NWs (Bierman et al., 2008; Zhu et al., 2008). The Eshelby twist has been suggested as the origin of a unique chiral microstructures grown by sponges (Zlotnikov et al., 2015), and as a method to create a variety of nanocrystals (Meng et al., 2013; Ren and Gao, 2014) including quantum supercrystals with optical chirality (Baimuratov et al., 2015). It is the Eshelby twist that makes the screw dislocation metastable within the NW; without it, the screw dislocation would be unstable in the NW due to the attraction to the NW side surface.

The dynamics and mechanics of Eshelby dislocations have been recently studied by atomistic (molecular dynamics) and continuum (dislocation dynamics) simulations as a deformation mode of NWs under torsion (Ryu et al., 2016; Weinberger and Cai, 2010b, 2012). The distributions of Eshelby dislocations has also been extensively studied recently (Berdichevsky, 2016; Le and Piao, 2016; Weinberger, 2011). However, the study on the stability of Eshelby 
dislocations against thermal fluctuation has been scarce, despite of its importance. Hirth and Frank (Hirth and Frank, 1958) considered the escape of a screw or mixed dislocation from the middle of an infinitely long wire using a continuum line tension model. The mean escape time was predicted as a function of NW radius, temperature, and shear modulus. However, this model made a number of simplifying assumptions that require a closer examination. First, a single line is used to represent the shape of the perfect dislocation. But dislocations in face-centered cubic (FCC) crystals dissociate into two partial dislocations bounding a stacking fault area, so that the dislocation core takes the shape of a ribbon. While the Eshelby potential well of two straight partial dislocations has been recently discussed (Roussel and Gailhanou, 2015), the study overestimates the stability of Eshelby dislocation because it only considers the escape of straight dislocations. Second, only the escape from the middle of the wire has been considered, while escape from the end of the wire may conceivably require less energy and hence be preferred. The effect of steps on the NW end surface, which is essential to the directional NW growth in the first place, was discussed only in a qualitative way, and requires further quantitative investigations.

In this work, the stability of Eshelby dislocations is investigated by combining atomistic and continuum models to address all the issues identified above. First, the energy barrier for the escape of Eshelby dislocations from FCC $\langle 110\rangle$ nanowires is predicted from an atomistic model as a function of NW radius, escape location, and surface step orientation. The dislocation naturally dissociates into partials in the atomistic model to minimize the energy. Furthermore, at the ends of the NW, the dislocation terminates with an extended node, where the dissociation width is greater than the equilibrium width in the middle. The escape from the end with the extended node is found to have a lower energy barrier than the escape from the middle of the NW. When the surface step is aligned with the dislocation's slip plane, the energy barrier for escape from the NW 
ends is reduced dramatically even further, making the dislocation more unstable inside the NW. A continuum line-tension model that accounts for partial dislocations is constructed, and is found to capture the main features of the atomistic predictions. We then apply the continuum model to predict the stability of Eshelby dislocations over a wider range of NW radii for FCC NWs, as well as for the PbSe (Zhu et al., 2008) and PbS (Bierman et al., 2008) NWs in which (non-dissociated) Eshelby dislocations have been observed.

\section{Methods}

\subsection{Atomistic Models}

Three FCC crystal NWs (Ni, Cu, Al) with circular cross sections are chosen as model systems for atomistic calculations to study the effect of modulus and stacking fault energy on the stability of Eshelby dislocations with embedded-atom model (EAM) potentials (Ercolessi and Adams, 1994; Mishin et al., 2001; Rao et al., 1999). We first prepare <110> NW of the perfect crystal with desired dimensions, and displace atoms according to the elasticity solution of a straight dislocation line along the cylinder axis with a perfect Burgers vector. The displacement field also leads to surface steps at the both ends of the NW. The structure is then relaxed by energy minimization, during which the perfect dislocation splits into two partial dislocations. Fig. 1(a) shows the relaxed atomic structure of the Eshelby dislocation in a $\mathrm{Ni}\langle 110\rangle \mathrm{NW}$ of diameter $D=$ $6.0 \mathrm{~nm}$. Since the twist angle per unit length, $\mathrm{b} /\left[\pi \mathrm{R}^{2}\right]$, is only about $8.8 \times 10^{-3}$ radian per nanometer, the twist of the nanowire is not easily recognized in Fig 1. The NW is subjected to zero-traction boundary conditions on its entire surface. The surface steps at the NW ends are chosen to be perpendicular to the dislocation's slip plane, so that they do not exert glide forces on the dislocation. Fig. 1(a) also shows that the dislocation terminates at a constricted node at the 
left end and an extended node at the right end of the NW. Such asymmetry has been reported earlier in threading dislocations in freestanding crystals (Leonardi et al., 2015; Rasmussen et al., 1997). It can be explained by the partial dislocations' preference to align along their partial Burgers vectors near the surface. We find that the constricted node at the left end is unstable during subsequent simulations, and thus artificially stabilize it by fixing a few atoms in the vicinity of the constricted node. Without this constraint, the NW can evolve into a state in which both ends are terminated with extended nodes split on two different $\{111\}$ planes with a cross-slip-like constriction in the middle of NW (Leonardi et al., 2015; Rasmussen et al., 1997), which would complicate our analysis. We expect that the constraint would not significantly change the energy barrier because it costs a negligible energy to move the cross-slip-like constriction along the nanowires (unless the constriction is very close to the end), and hence the constraint would hardly affect the saddle point configuration for the escape mode from the middle. The energy barrier for escape is computed by a modified free-end version of the string method (Ryu et al., 2011) which searches for the lowest energy barrier path from the initial metastable configuration in Fig 1(a) toward the final configuration in Fig 1(e) beyond the saddle point. The string method optimizes a continuous reaction pathway (string), i.e. the chain of atomic configurations, connecting the initial and the final states, to a minimum energy path. During the optimization, the neighboring configurations are kept equally spaced by repositioning the configurations along the path at each iteration step. By keeping the total length of the string during the optimization, we can prevent the free-end final state (such as Fig 1(e)) from sliding far away from the saddle point. In this way, an adequate discretization of the chain can near the saddle point be achieved without an excessive number of configurations along the chain, thus significantly reducing the computational cost. A 
climbing image method (Henkelman et al., 2000) is subsequently used to precisely locate the saddle configuration in Fig 1(c).

\subsection{Line Tension Model}

In order to predict the energy barrier for Eshelby dislocation escape from NWs with larger diameters and for materials where a reliable interatomic-potential is not yet available, we develop a continuum line tension model along the line of Hirth and Frank (Hirth and Frank, 1958), but accounting for the dissociation of dislocations into partials. The dislocation is represented by two functions, $\mathrm{y}_{1}(x)$ and $\mathrm{y}_{2}(x)$, for the shapes of the two partial dislocations. The energy of the NW is a functional of $\mathrm{y}_{1}(x)$ and $\mathrm{y}_{2}(x)$ with the following expression.

$$
\begin{aligned}
& \mathrm{E}\left[\mathrm{y}_{1}(x), y_{2}(x)\right]=\gamma_{S F} \int_{x_{i}}^{x_{f}}\left|y_{2}(x)-y_{1}(x)\right| d x \\
& +\int_{x_{i}}^{x_{f}}\left(E_{c}\left(\tan ^{-1} \frac{d y_{1}}{d x}-\frac{\pi}{6}\right) \sqrt{1+\left(\frac{d y_{1}}{d x}\right)^{2}}+E_{c}\left(\tan ^{-1} \frac{d y_{2}}{d x}+\frac{\pi}{6}\right) \sqrt{1+\left(\frac{d y_{2}}{d x}\right)^{2}}\right) \mathrm{dx} \\
& +\int_{x_{i}}^{x_{f}} U\left(y_{1}(x), y_{2}(x)\right) \mathrm{dx}+\frac{1}{2} \mathrm{E}_{\text {step }}\left(\left|y_{1}\left(x_{i}\right)\right|+\left|y_{1}\left(x_{f}\right)\right|+\left|y_{2}\left(x_{i}\right)\right|+\left|y_{2}\left(x_{f}\right)\right|\right)
\end{aligned}
$$

The $x$-axis is chosen to be the NW axis. All integrals are performed along the length of the NW. The first term is the energy of the stacking fault energy, where $\gamma_{\mathrm{SF}}$ is the stacking fault energy per unit area. The equilibrium separation between the two partial dislocations in the bulk is $l_{0}=A$ / $\gamma_{\mathrm{SF}}$ where $\mathrm{A}=\left(\frac{1}{4}-\frac{1}{12(1-v)}\right) \frac{\mu b^{2}}{2 \pi}, \mu$ is the shear modulus, $v$ is Poisson's ratio and $\mathrm{b}=\frac{\mathrm{a}}{\sqrt{2}}$ is the magnitude of the Burgers vector of the perfect dislocation. The second term represents the line energy of the two partials, where $E_{\mathrm{c}}(\theta)$ is the orientation dependent line energy per unit length, and $\theta$ is the character angle between line orientation and the partial Burgers vector. We use the 
expression $\mathrm{E}_{\mathrm{c}}(\theta)=\frac{\mu b_{p}^{2}}{4 \pi} \ln \left(\frac{R}{r_{0}}\right)\left(\cos ^{2} \theta+\frac{\sin ^{2} \theta}{1-v}\right)$ to account for the higher energy of the edge orientation $(\theta=\pi / 2)$ than that of the screw orientation $(\theta=0)$, where $b_{p}$ and $r_{0}$ are the Burgers vector and the core radius of a partial dislocation, respectively. All input parameters are obtained from atomistic calculations, as shown in Table $\mathrm{I}$, except the core radius, $\mathrm{r}_{0}$. In Appendix A, we discuss how well the atomistic potentials reproduce various materials properties. The third term represents the elastic energy due to the confinement effects of the Eshelby twist, surface attraction from image stress, and the elastic interaction between two partial dislocations. We derived this expression starting from the known stress field expressions of edge and screw dislocations in a cylinder (Eshelby, 1979). The stress field allows us to obtain the interaction forces on the partial dislocations. Finally, we construct an energy function $\mathrm{U}\left(\mathrm{y}_{1}, \mathrm{y}_{2}\right)$ such that its negative derivative with respect to $\mathrm{y}_{1}$ and $\mathrm{y}_{2}$ match the interaction forces. The energy function was derived as a function of the central position and the separation distance of two partial dislocations based on the similar method in a previous study (Roussel and Gailhanou, 2015). Here, the so called "local density approximation" is used because the expression

$$
\begin{aligned}
U\left(y_{1}, y_{2}\right)=- & \frac{\mu b_{s}^{2}\left(-2 R^{2}+y_{1}^{2}+y_{2}^{2}\right)^{2}}{4 \pi R^{4}} \\
& +\frac{\mu b_{s}^{2}}{2 \pi}\left(-\ln \left|y_{1}-y_{2}\right|+\frac{\ln \left(R^{2}-y_{1}^{2}\right)}{2}+\frac{\ln \left(R^{2}-y_{2}^{2}\right)}{2}+\ln \left(R^{2}-y_{1} y_{2}\right)\right) \\
& +\frac{\mu b_{e}^{2}}{2 \pi(1-v)}\left(\ln \left|y_{1}-y_{2}\right|+\frac{\ln \left(R^{2}-y_{1}^{2}\right)}{2}+\frac{\ln \left(R^{2}-y_{2}^{2}\right)}{2}\right. \\
& \left.-\ln \left(R^{2}-y_{1} y_{2}\right)-\frac{R^{2}\left(y_{1}-y_{2}\right)^{2}}{2\left(y_{1} y_{2}-R^{2}\right)^{2}}\right)
\end{aligned}
$$

is valid, strictly speaking, for perfectly straight dislocations at offsets $\mathrm{y}_{1}$ and $\mathrm{y}_{2}$ from the NW axis, while we use the $U\left(y_{1}, y_{2}\right)$ to represent the local energy density (per unit length) of a (smoothly) 
curved dislocation. This is a major assumption in the line tension model and has been used in Hirth and Frank. It is worth noting that, instead of directly modelling the twist in the line tension model, its effect is taken into account indirectly in the energy function. Hence, the energy barriers for the nanowire with very large twist angle (i.e. very small diameter) may not be accurate. The validity of this and other approximations in the line tension model is tested by comparing its predictions with the atomistic data, as demonstrated in the following sections. The fourth term is the contribution from the step energy at two ends of nanowires where $E_{\text {step }}$ represent the step energy when the height of surface step is same as the magnitude of the perfect Burgers vector, $\frac{\mathrm{a}}{2}\langle 110\rangle$. Since the height of the step formed by a partial dislocation sweep is half of the perfect Burgers vector, we approximate the step energy as $\frac{1}{2} E_{\text {step }}$.

The shape of the partial dislocation pairs at the metastable phase (Fig 1(b)) can be predicted by finding two functions $\mathrm{y}_{1}(x)$ and $\mathrm{y}_{2}(x)$ which minimize the energy functional given as Eq (1). Because the analytic solution is not available, we solve the equation numerically by describing the dislocation as the line segments connecting the nodes regularly spaced along the axial direction (x-axis) (Kang et al., 2012). The minimum energy paths for dislocation escape with various mechanisms are obtained using a modified version of the string method, similar to that used in the atomistic model. The string method applied to the continuum model is essentially the same as that applied to the atomistic model. The only difference is that when applied to the atomistic model, each configuration along the chain is represented by the atomic coordinates, but when applied to the continuum model, each configuration along the chain is represented by an array describing the (discretized) shape of the perfect dislocation line, or two arrays for the two partial dislocation lines. Specifically, the shape of the dislocation line $y(x)$ is discretized by $\left\{y_{i}\right\}$, where $y_{i}=y(x i)$, with $x_{i}=$ i $\Delta \mathrm{x}, \Delta \mathrm{x}=0.1 \mathrm{~nm}$ for perfect dislocations and $\Delta \mathrm{x}=0.3 \mathrm{~nm}$ for partial dislocations 
(a)

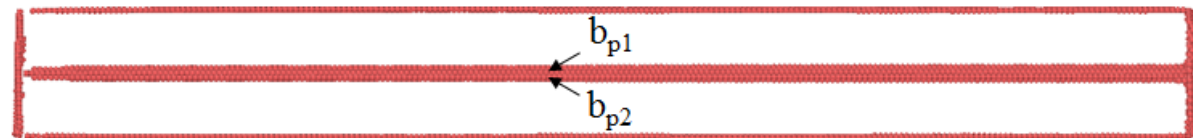

(b)

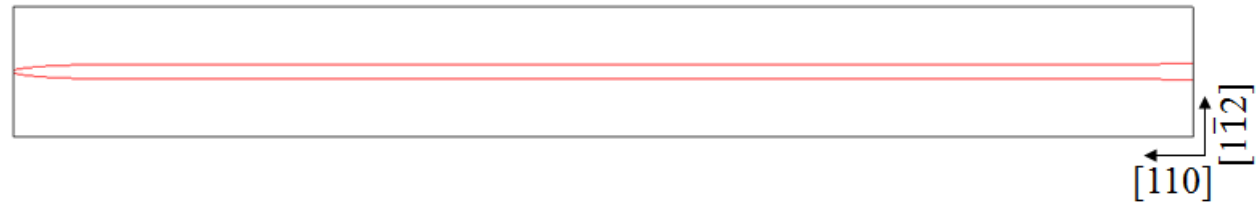

(c)

(d)

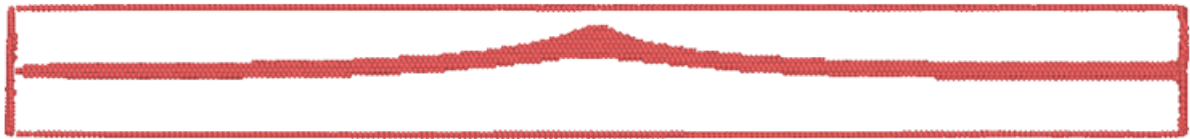

)

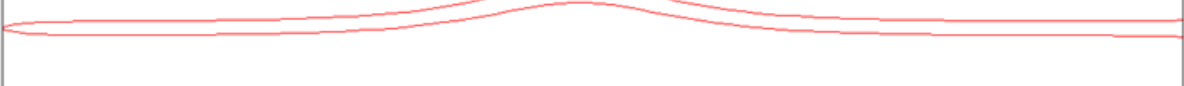

(e)

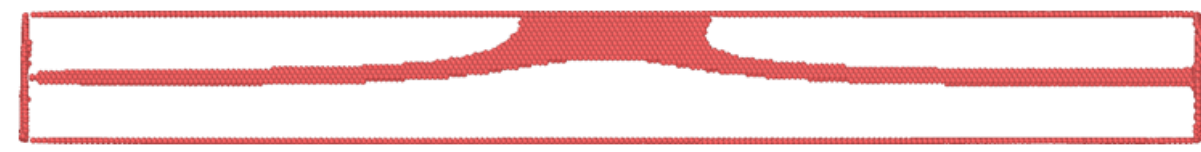

(f)

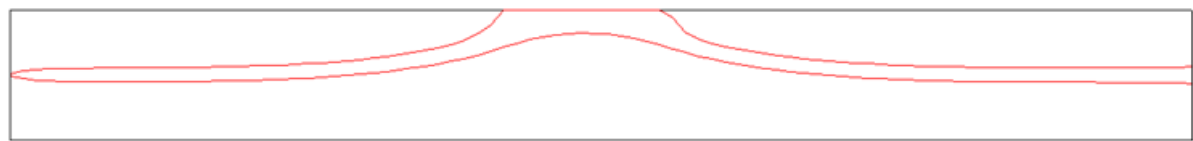

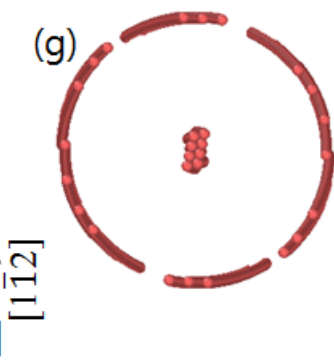

(h)
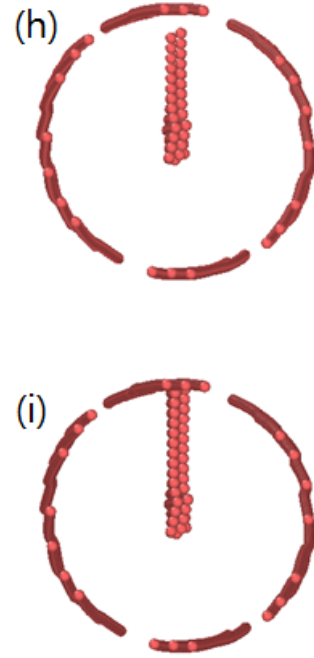

Figure 1. Equilibrium structure of the Eshelby screw dislocation inside a $\mathrm{Ni}\langle 110\rangle \mathrm{NW}$ with diameter $D=6.0 \mathrm{~nm}$ in (a) atomistic model and (b) continuum line tension model. The surface step at the NW ends are perpendicular to the dislocation's slip plane, i.e. on the (11̄2) plane. Saddle-point configurations for dislocation escape from the middle of the NW in (c) atomistic model and (d) continuum model. Unstable configuration in (e) atomistic model and (f) continuum model. The side views of atomistic models for equilibrium (g), saddle (h), and unstable (i) configurations shows the dislocation core structure as well as the twist of the nanowire. In (a), (c), and (e), atoms with central symmetry parameters exceeding a threshold are selectively plotted to show the dislocation core and some surface atoms. 
Table I. Input parameters of the continuum line tension model and predictions of critical NW diameter for Eshelby dislocation escape for different materials. The input parameters include shear modulus, Poisson's ratio, stacking fault energy, and step energy. Stacking fault energy $\gamma_{\mathrm{SF}}$, and step energy $\mathrm{E}_{\mathrm{step}}$ are directly obtained from atomistic calculations with the EAM and FS potentials (Ackland and Thetford, 1987; Ercolessi and Adams, 1994; Mishin et al., 2001; Rao et al., 1999), and the effective shear modulus $\mu$ and Poisson's ratio $v$ are obtained from anisotropic elastic constants by the approach of Scattergood and Bacon (Scattergood, 1982; Scattergood and Bacon, 1975) which matches the energy prefactors of edge and screw dislocations for a given slip system. The elastic constants of $\mathrm{PbSe}$ and $\mathrm{PbS}$ are experimental measurements (Madelung, 1983), while others are obtained by atomistic calculations.

\begin{tabular}{|c|c|c|c|c|c|c|c|c|c|c|}
\hline & $\begin{array}{c}\mathrm{C}_{11} \\
(\mathrm{GPa})\end{array}$ & $\begin{array}{c}\mathrm{C}_{12} \\
(\mathrm{GPa})\end{array}$ & $\begin{array}{c}\mathrm{C}_{44} \\
(\mathrm{GPa})\end{array}$ & $\begin{array}{c}\mu \\
(\mathrm{GPa})\end{array}$ & $v$ & $\begin{array}{c}\gamma_{\mathrm{SF}} \\
\left(\mathrm{mJ}^{2}\right)\end{array}$ & $\begin{array}{c}\mathrm{E}_{\mathrm{step}} \\
(\mathrm{eV} / \mathrm{A})\end{array}$ & $\begin{array}{c}\mathrm{D}_{\mathrm{c}}(\mathrm{nm}) \\
\begin{array}{c}\mathrm{M}-m o d e \\
(\text { atomistic })\end{array}\end{array}$ & $\begin{array}{c}\mathrm{D}_{\mathrm{c}}(\mathrm{nm}) \\
\begin{array}{c}\text { E-mode } \\
(\text { atomistic) }\end{array}\end{array}$ & $\begin{array}{c}\mathrm{D}_{\mathrm{c}}(\mathrm{nm}) \\
\mathrm{E} \text { Eparallel }\end{array}$ \\
\hline $\mathrm{Al}$ & 118 & 63 & 36.5 & 31.8 & 0.342 & 120.7 & 0.101 & $\begin{array}{c}3.0 \\
(4.5)\end{array}$ & $\begin{array}{c}2.8 \\
(5.0)\end{array}$ & 17.0 \\
\hline $\mathrm{Cu}$ & 169 & 122 & 75.3 & 42.1 & 0.321 & 44.6 & 0.129 & $\begin{array}{c}3.5 \\
(5.7)\end{array}$ & $\begin{array}{c}5.0 \\
(7.3)\end{array}$ & 27.6 \\
\hline $\mathrm{Ni}$ & 245 & 149 & 120 & 75.6 & 0.374 & 119.5 & 0.205 & $\begin{array}{c}2.7 \\
(4.1)\end{array}$ & $\begin{array}{c}3.3 \\
(4.8)\end{array}$ & 15.2 \\
\hline $\mathrm{PbSe}$ & 123.7 & 19.3 & 15.9 & 15.9 & 0.590 & $\mathrm{~N} / \mathrm{A}$ & $\mathrm{N} / \mathrm{A}$ & $16^{\mathrm{a}}$ & & \\
\hline $\mathrm{PbS}$ & 124 & 33 & 23 & 23 & 0.492 & N/A & N/A & $\begin{array}{c}0^{\mathrm{a}, \mathrm{b}} \\
(15)^{\mathrm{c}}\end{array}$ & & \\
\hline
\end{tabular}

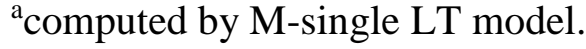

benergy barrier is zero for the mixed dislocation observed in experiments (Bierman et al., 2008) in the absence of the lattice resistance which is not considered in this study.

'energy barrier if the dislocation in $\mathrm{PbS}$ nanowire is a pure screw dislocation 


\section{Results}

\subsection{Energy barriers obtained by atomistic simulations}

Following Hirth and Frank (Hirth and Frank, 1958), we first consider the escape of Eshelby dislocation from the middle of the NW. We will refer to this escape mode as the M-mode in the following. Fig. 1(c) shows the saddle-point configuration and Fig. 1(e) shows the unstable configuration beyond the saddle point. In Fig. 1 (c) and (e), the middle section of the dislocation line bows out from the NW axis, locally untwisting the NW. The NW length has been chosen to be much longer than the bowed out region of the dislocation to ensure that the results are representative of an infinite long NW considered by Hirth and Frank.

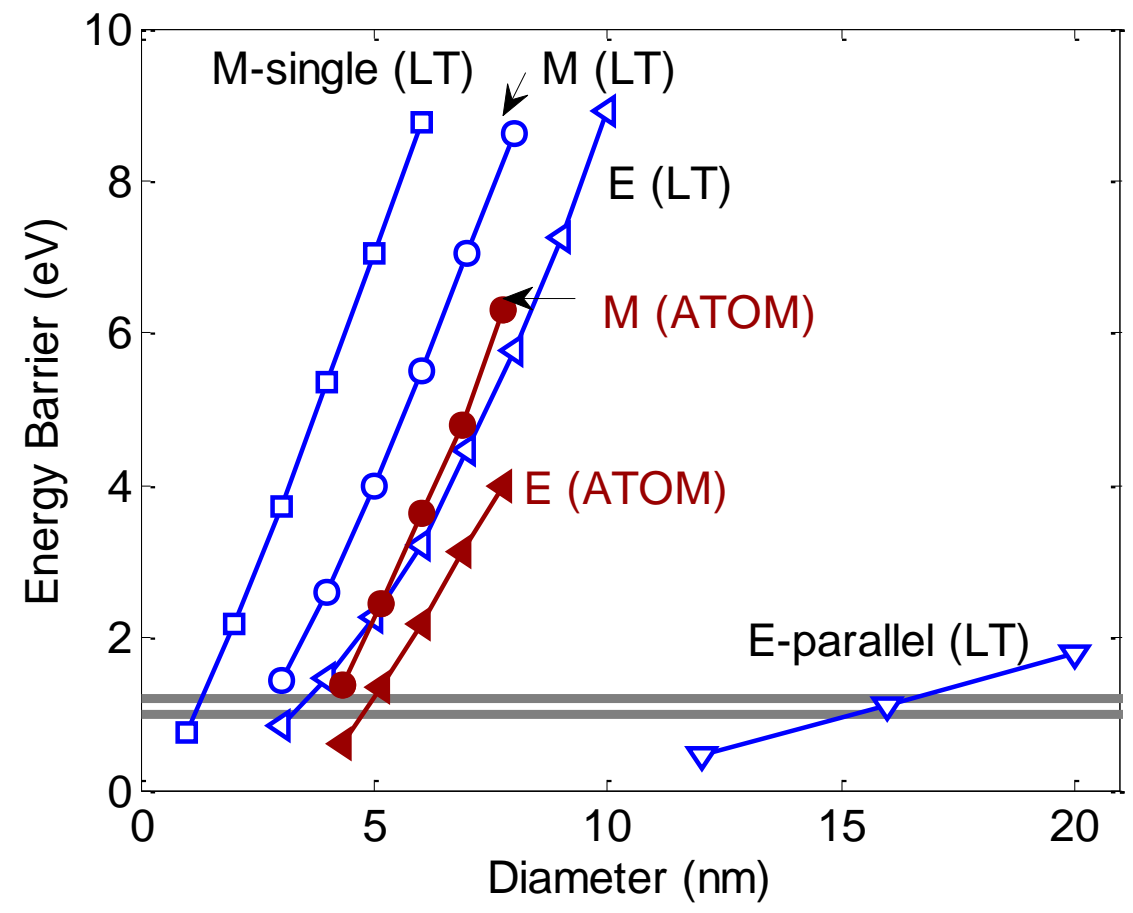

Figure 2. Filled symbols: energy barrier predicted by atomistic model (ATOM) for Eshelby dislocation escape from $\mathrm{Ni}\langle 110\rangle \mathrm{NW}$ as a function of NW diameter $D$ for different escape modes: from middle of NW (M-mode), from NW end with extended node (Emode). Open symbols: energy barrier predicted by continuum line tension (LT) model for different modes: M, E, E-parallel, M-single (see text). Two gray horizontal lines at $1.2 \mathrm{eV}$ and $1.0 \mathrm{eV}$ are drawn to indicate the threshold energy barrier for M-mode and E-mode, 
respectively. The surface step is perpendicular to the dislocation glide plane for all data points except for the case of E-parallel, in which the surface step is parallel to the glide plane.

Fig 2 shows the energy barrier as a function of NW radius. As the nanowire diameter increases, the energy barrier increases rapidly. Roughly speaking, an escape event can occur over the experimental time scale ( $\sim 10^{3}$ seconds) at room temperature if the energy barrier $E_{\mathrm{b}}$ is less than $\sim 1.2 \mathrm{eV}$. The escape time $\tau$ can be estimated as $1 / f$ where the escape frequency $f$ is given as $f=\mathrm{N} f_{0} \exp \left(-\frac{E_{b}}{k_{B T}}\right)$. Assuming a NW length of $L=1 \mu \mathrm{m}$, the number of nucleation cite $\mathrm{N}$ can be estimated as $\mathrm{L} / \mathrm{b}_{\mathrm{p}}$ and the attempt frequency $f_{0}$ is assumed to be around the Debye frequency, $\sim 10^{13} \mathrm{~Hz}$. Based on this analysis, if only M-mode of escape were allowed, the Eshelby dislocation would remain stable almost indefinitely in Ni NWs with diameters $D \geq 4 \mathrm{~nm}$.

However, it is conceivable that the dislocation may prefer to escape from the NW end, where the energy barrier may be lower because the dislocation only needs to form half the bend than that required for escaping from the middle. Fig. 3(a), (c), and (e) show the evolution of the dislocation shape for escaping from the NW end with an extended node. The energy barriers for escaping from the end with extended node (E-mode) as functions of NW diameter are also plotted in Fig. 2. The escape from the NW end with extended node has lower energy barrier than the energy barrier from the escape from the NW middle, as expected. Although the dislocation only forms half the bend when escaping by the E-mode, the energy barrier is higher than half of the energy barrier for the M-mode.

At the intersection point where a screw dislocation terminates at the surface, it must be connected to a surface step. The tendency to reduce the step energy (by shortening the length of the surface step) leads to a force on the dislocation. So far we have let the surface step be 
perpendicular to the dislocation glide plane. This has a stabilizing effect on the dislocation, because whenever the dislocation moves away from the NW center, more surface step is created, increasing the energy, as illustrated in Fig. 3(i). The step energy increases the energy barrier for the E-mode but not the M-mode (for which no step is created), which may explain why the energy barrier for the E-mode is more than half of that for the M-mode.

(a)

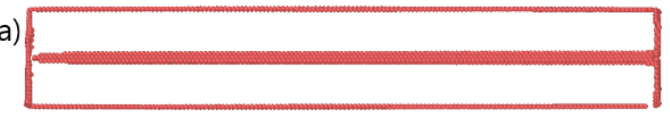

(c)

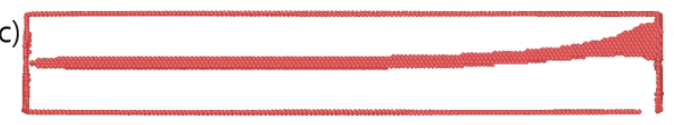

(e)
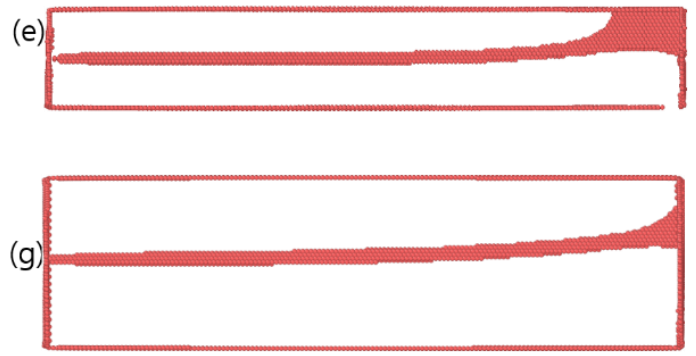

(b)

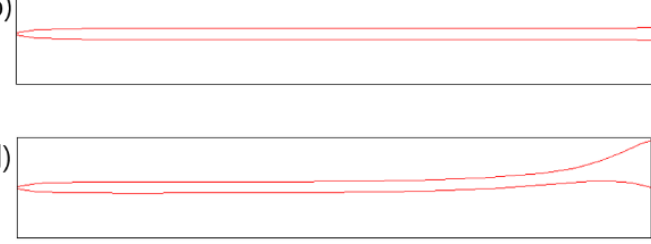

(f)
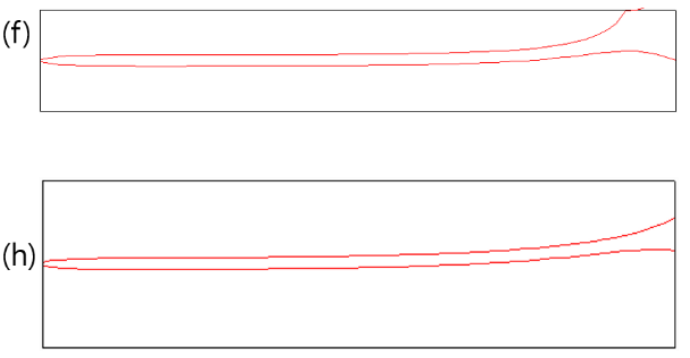

(j)

(i)
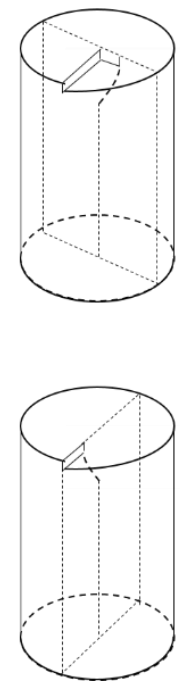

Figure 3. Equilibrium structure of the Eshelby screw dislocation inside an $\mathrm{Ni}\langle 110\rangle \mathrm{NW}$ with diameter $D=6.0 \mathrm{~nm}$ in (a) atomistic model and (b) continuum line tension model. The surface step at the NW ends are perpendicular to the dislocation's slip plane. Saddlepoint configurations for dislocation escape from the end of the NW with extended node in (c) atomistic model and (d) continuum model. Unstable configuration in (e) atomistic model and (f) continuum model. In (a)-(f), the surface step is perpendicular to dislocation's slip plane, as illustrated in (i). Equilibrium structure of the Eshelby screw dislocation with diameter $D=10.3 \mathrm{~nm}$ when the surface step is parallel to dislocation's slip plane in $(\mathrm{g})$ atomistic model and (h) continuum model, with the surface step illustrated in (j).

To enable a fair comparison between the two escape modes, we need to account for the fact that escaping by the E-mode can only occur at the ends of the NW while escaping by the Mmode can occur anywhere along the NW length. In other words, there are more nucleation sites for M-mode than E-mode. For a typical $1.5 \mu \mathrm{m}$ long nanowire grown in experiments, the number 
of nucleation sites for the E-mode is 2 while the number of the nucleation sites for the M-mode is about $15,000 / \frac{3.52}{\sqrt{2}} \approx 6 \times 10^{3}$ (which is about a factor of $3 \times 10^{3}$ greater than that for the E-mode) Then, at room temperature the escape rate of the two modes would equal if the energy barrier of the E-mode is about $0.2 \mathrm{eV}$ lower than that of the M-mode. Given that we have estimated the threshold energy barrier for the M-mode to be $\sim 1.2 \mathrm{eV}$, the threshold energy barrier for the Emode is estimated to be $\sim 1.0 \mathrm{eV}$. The critical diameter below which the dislocation becomes unstable at the experimental time scale can be estimated by finding the intersecting point between the energy curve and the horizontal threshold energy lines, as shown in Fig 2. We can see that for NW diameters greater than $5 \mathrm{~nm}$, the dislocation is stable against both modes of escape. Due to the significantly lower energy barrier for E-mode, we conclude that the more favorable mode of escape is from the end of the NW. This conclusion applies to all three FCC metals ( $\mathrm{Ni}, \mathrm{Cu} \mathrm{Al})$ considered in this study, as depicted in Fig $\mathbf{4}$ for $\mathrm{Cu}$ and $\mathrm{Al}$. We note that the energy barrier difference between $\mathrm{M}$-mode and $\mathrm{E}$-mode is much smaller in $\mathrm{Al}$ than in $\mathrm{Ni}$ and $\mathrm{Cu}$, because of the high stacking fault energy in $\mathrm{Al}$, making the dislocation core not very extended. 

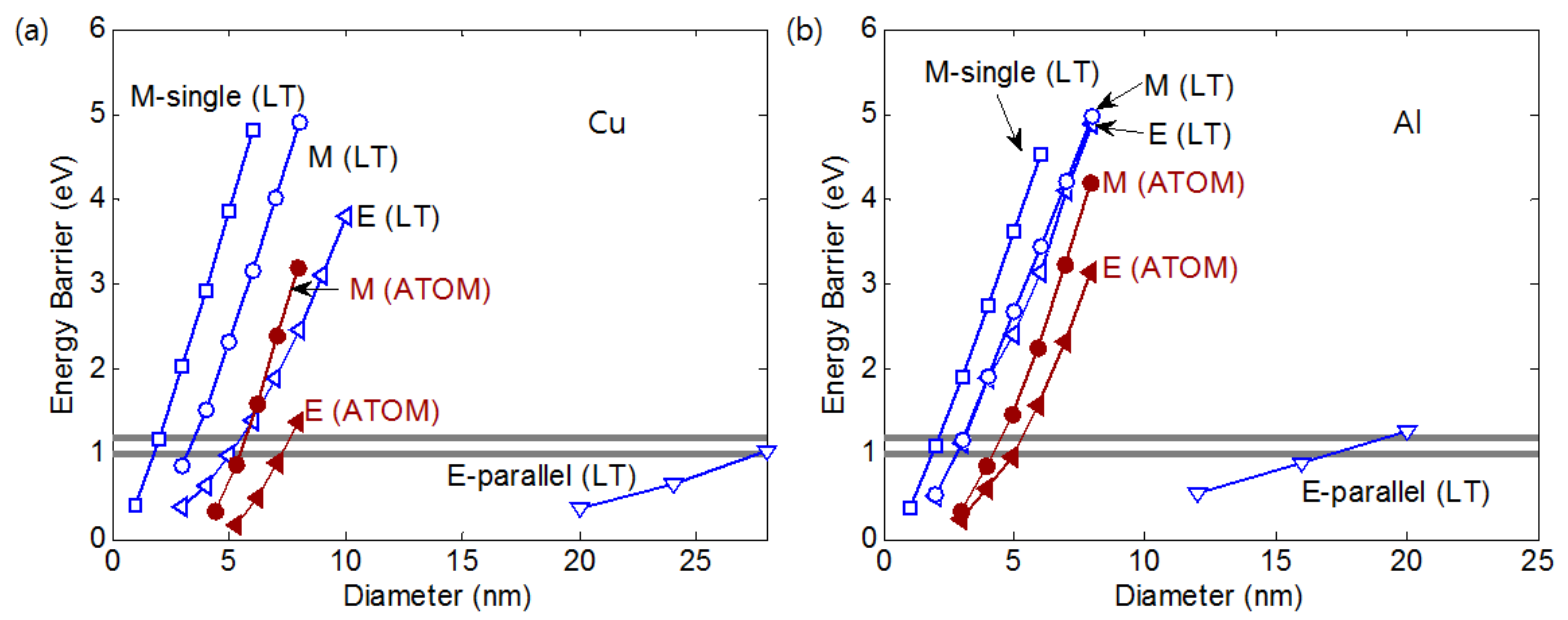

Figure 4. Solid lines: energy barrier predicted by atomistic model (ATOM) for Eshelby dislocation escape from (a) $\mathrm{Cu}$ and (b) $\mathrm{Al}\langle 110\rangle \mathrm{NWs}$ as functions of NW diameter $D$ for different escape locations: middle of NW (M-mode), NW end with extended node (Emode). Dashed lines: energy barrier predicted by continuum line tension model (LT) for M-mode, E-mode, E-parallel, and M-single (perfect dislocation) Two dotted horizontal lines represent $1.0 \mathrm{eV}$ and $1.2 \mathrm{eV}$ which are threshold energy barrier for E-mode and Mmode, respectively. For all data points, surface step is perpendicular to dislocation's slip plane. $\mathrm{Cu}$ shows lower energy barrier at small diameter due to its smaller stacking fault energy, while energy barrier increases faster as diameter increases due to larger shear modulus.

All the results discussed so far correspond to the case where the surface step is perpendicular to the dislocation slip plane. The situation is very different if the surface step is parallel to the slip plane, which will be referred as E-parallel mode. This situation is perhaps rare to find for NWs grown assisted by the screw dislocation, because the surface steps is likely to have a spiral shape at the end of the NW. Yet, this is the case if the screw dislocation is formed by glide during the twisting a previously defect-free NW(Weinberger and Cai, 2010a). In any case, this situation can be considered as a lower bound for the energy barrier, i.e. the most conservative estimate of the thermal stability. In this case, dislocation motion (in the favorable direction) reduces the length of the surface step, thus lowering the energy, as illustrated in Fig. 3(j). The 
force exerted by the surface step alters the equilibrium shape of the dislocation in the metastable state, as shown in Fig. 3(g). In this case, E-parallel escape has vanishingly small energy barrier ( $0 \mathrm{eV}$ ) for Ni NW of $D=10 \mathrm{~nm}$ (the maximum size considered in the atomistic study limited by computational resources). This is understandable because the dislocation node is rather close to the NW surface even in the equilibrium (metastable) state and the surface step energy reduces even further as the dislocation escapes. Therefore, for surface step parallel to dislocation's slip plane, we predict the the Eshelby dislocation is unstable in the Ni NW at the experimental time scale even if the NW diameter is as large as $10 \mathrm{~nm}$. The critical diameter at which the Eshelby dislocation becomes stable (at the experimental time scale) must be larger than $10 \mathrm{~nm}$, and should preferably be predicted by a different approach due to the high computational cost of the atomistic model.

\subsection{Energy barriers obtained by the line tension model}

The equilibrium shape of the dislocation for Ni predicted by the line tension model is shown in Fig. 1(b), for the case of surface step non-parallel to the slip plane. The input parameters for the line tension model are listed in Table I. The evolution of the dislocation shape for M-mode escape is shown in Fig. 1(b), (d), and (f). The evolution for the E-mode escape (surface step perpendicular to slip plane) is shown in Fig. 3(b), (d), and (f). The constricted node at one end and extended node at the other end are successfully captured, which is caused by the orientation dependence of the line energy $E_{\mathrm{c}}(\theta)$. The equilibrium shape of the dislocation when the surface step is parallel to the slip plane is shown in Fig. 3(h). In all cases, the line tension predictions resemble the atomistic predictions remarkably well. The difference between M-mode and E-mode is captured by the LT very well. 
Fig. 2 compares the predictions of the energy barriers from the line tension model against the atomistic model, for various escape modes. We find that the line tension model with core radius $\mathrm{r}_{0}=b_{p}$ reproduces well the dislocation configuration from atomistic simulations. There are no other adjustable parameters in the line tension model. In M-mode, the line tension model reproduces the diameter dependence of the energy barrier predicted by the atomistic model remarkably well with a constant offset. The offset is somewhat expected because the line tension is a crude approximation of the non-linear interatomic interactions at the dislocation core. A similar offset has also been observed in the previous study on the cross-slip(Kang et al., 2014). This agreement with the atomistic data confirms the validity of the various approximations in the line tension model. The line tension model also successfully predicts the lower energy barrier for escape from the extended node (E-mode), using the surface step energy directly computed from the atomistic model, i.e. without any more adjustable parameters. The difference in the energy barrier between the M-mode and E-mode predicted by the line tension model agrees well with the atomistic data.

The agreements observed above establish the line tension model as a useful tool to make predictions at NW sizes beyond the reach of atomistic models. For example, we can predict the energy barrier for E-mode escapes when the surface step is parallel to the slip plane. The critical NW diameter corresponding to $\mathrm{E}_{\mathrm{b}}=1.0 \mathrm{eV}$ is predicted to be $D=16 \mathrm{~nm}$, which would be very cumbersome to handle within a full atomistic simulation. Due to the overestimation of energy barrier by line tension model, the critical radius from atomistic model is expected to be larger than the predicted values by 1 or $2 \mathrm{~nm}$.

We then compare our prediction on the stability of $\mathrm{Cu}$ NWs with experiments on the screwdislocation-driven growth of Cu NWs (Meng, 2012). As shown in Table I, our model predicts that 
the critical diameter is $7.3 \mathrm{~nm}$ at $300 \mathrm{~K}$ (corresponding to $1.2 \mathrm{eV}$ for E-mode). Even at the growth temperature (333 K) (Meng, 2012), where the threshold energy barrier is $1.33 \mathrm{eV}$, the critical diameter (for E-mode) becomes $7.5 \mathrm{~nm}$, which is significantly smaller than the diameters of $\mathrm{Cu}$ NWs in experiments, 45-100 nm (Meng, 2012). Even if we consider the E-parallel mode of escape, the critical diameter becomes $27.6 \mathrm{~nm}$, which is still lower than the diameters of $\mathrm{Cu}$ NWs in the experiments. Therefore, our predictions are consistent with the observation that Eshelby screw dislocation is stable in $\mathrm{Cu}$ NWs of diameters of 45-100 nm.

\section{Discussion}

Having established the validity of the line tension approaches, we computed the energy barrier for the escape of a perfect (non-dissociated) dislocation, with core radius $r_{0}=b$ to test the importance of considering the dissociation of dislocations into partials. The energy expression for single dislocation is identical to the original Hirth and Frank work, but we added an orientationdependent line tension to compute the energy barrier (referred to as the M-single model). In Hirth and Frank, the energy barrier of the single dislocation escape has been calculated by assuming a triangular dislocation shape, as explained in detail in Appendix B. 
(a)

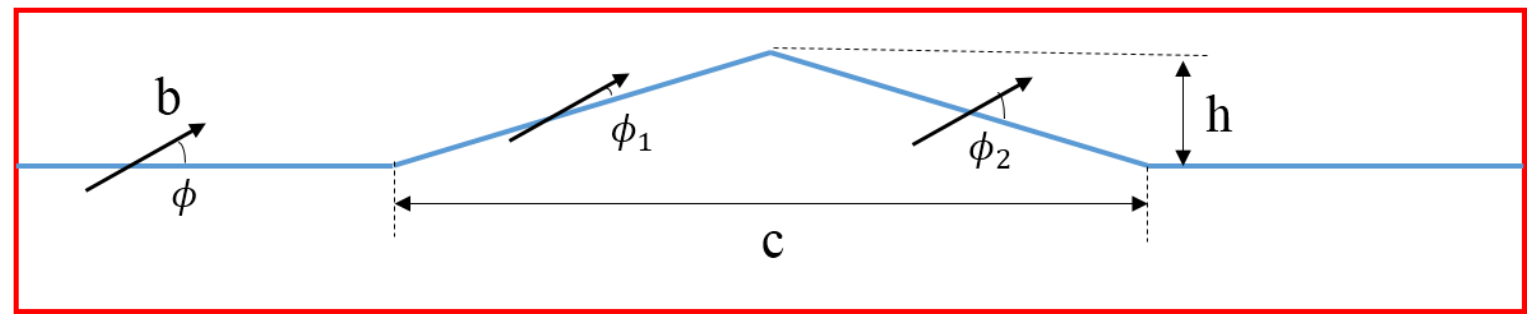

(b)

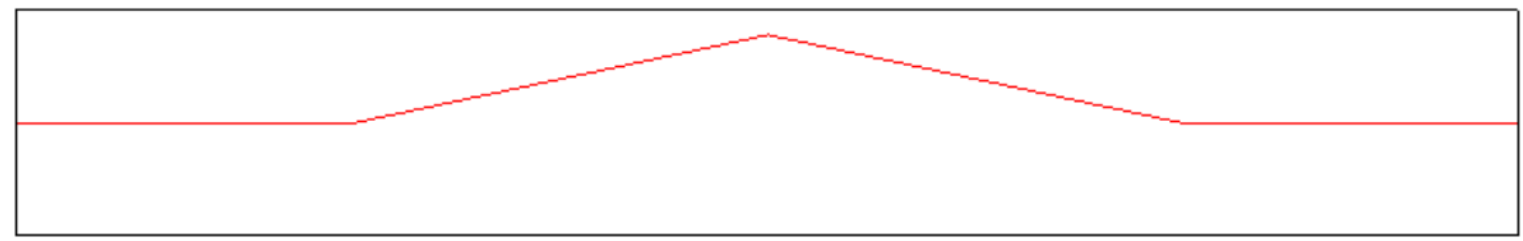

(c)

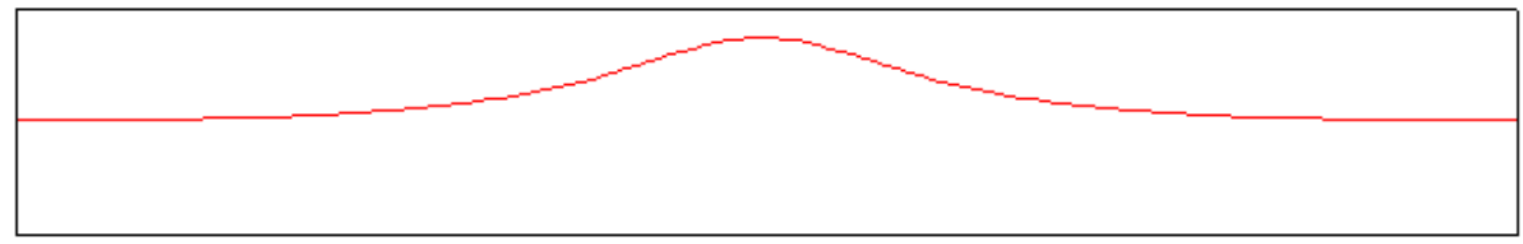

Figure 5. (a) Schematic of the triangular dislocation studied in the Hirth and Frank. The saddle point configuration of $\mathrm{Cu} N W$ with diameter $\mathrm{D}=3.0 \mathrm{~nm}$ with (b) the modified Hirth and Frank model (Eq. (S6)) and (c) the line tension model for a perfect single dislocation (M-single). For the same energy function given in S6, the modified Hirth and Frank model gives the optimal $\mathrm{h}$ and $\mathrm{c}$ values (Table S2) with a constrained dislocation shape, while the line tension model gives the optimal dislocation geometry. Still, the energy barrier for two models are quite similar, $2.17 \mathrm{eV}$ and $2.03 \mathrm{eV}$, respectively.

Escape energy barriers from the original Hirth-Frank (H-F), the modified H-F, M-single, M-mode are presented in Table 2 for $\mathrm{Cu}$ NW. By ignoring the orientation dependence, the original H-F predicts significantly lower energy barriers than the modified H-F. The modified H-F and Msingle models use the same energy functional, but the M-single model assumes a curved dislocation shape. Hence, energy barrier from M-single is slightly lower than the modified H-F, which assumes a triangular dislocation shape. The M-mode model assumes two curved partial dislocations, predicting the lowest energy barrier, as expected. 
Table 2. The energy barrier obtained by the original Hirth and Frank (H-F) equation, Eq. (S4), the modified Hirth and Frank equation Eq. (S6), line tension model for M-single, and line tension model for M-mode for $\mathrm{Cu}$ NWs. We use $\mathrm{r}_{0}=b$ for all calculations.

\begin{tabular}{|c|c|c|c|c|c|c|c|c|}
\hline \multirow{2}{*}{$\mathrm{D}(\mathrm{nm})$} & \multicolumn{3}{|c|}{ Original H-F } & \multicolumn{3}{c|}{ Modified H-F } & M-single & M-mode \\
\cline { 2 - 9 } & $\mathrm{h}^{*}(\mathrm{~nm})$ & $\mathrm{c}^{*}(\mathrm{~nm})$ & $\mathrm{E}_{\mathrm{b}}(\mathrm{eV})$ & $\mathrm{h} *(\mathrm{~nm})$ & $\mathrm{c}^{*}(\mathrm{~nm})$ & $\mathrm{E}_{\mathrm{b}}(\mathrm{eV})$ & $\mathrm{E}_{\mathrm{b}}(\mathrm{eV})$ & $\mathrm{E}_{\mathrm{b}}(\mathrm{eV})$ \\
\hline 1 & 0.388 & 1.538 & 0.32 & 0.388 & 2.174 & 0.45 & 0.41 & 0 \\
\hline 3 & 1.164 & 7.826 & 1.55 & 1.164 & 10.937 & 2.17 & 2.03 & 0.50 \\
\hline 5 & 1.940 & 14.883 & 2.94 & 1.940 & 20.736 & 4.11 & 3.86 & 1.52 \\
\hline
\end{tabular}

As illustrated in Fig. 2, the energy barrier for non-dissociated perfect dislocations is significantly higher than that of dissociated dislocations. This difference is caused by several reasons. First, the line tension of dislocation is proportional to the Burgers vector. Because $b_{p}^{2}=$ $\frac{1}{3} b^{2}$, the energy cost for bending dislocation is significantly larger for the perfect dislocation than the sum of two partial dislocations. Second, because of the splitting, partial dislocations located nearer to the NW surface where the image force toward surface is stronger. Third, partial dislocations offset from the NW axis also generates less Eshelby twist, and hence a shallower energy well for the dislocations. Thus, we conclude that the consideration of dislocation dissociation into partials is essential in the prediction of the stability of Eshelby dislocation in FCC NWs. 


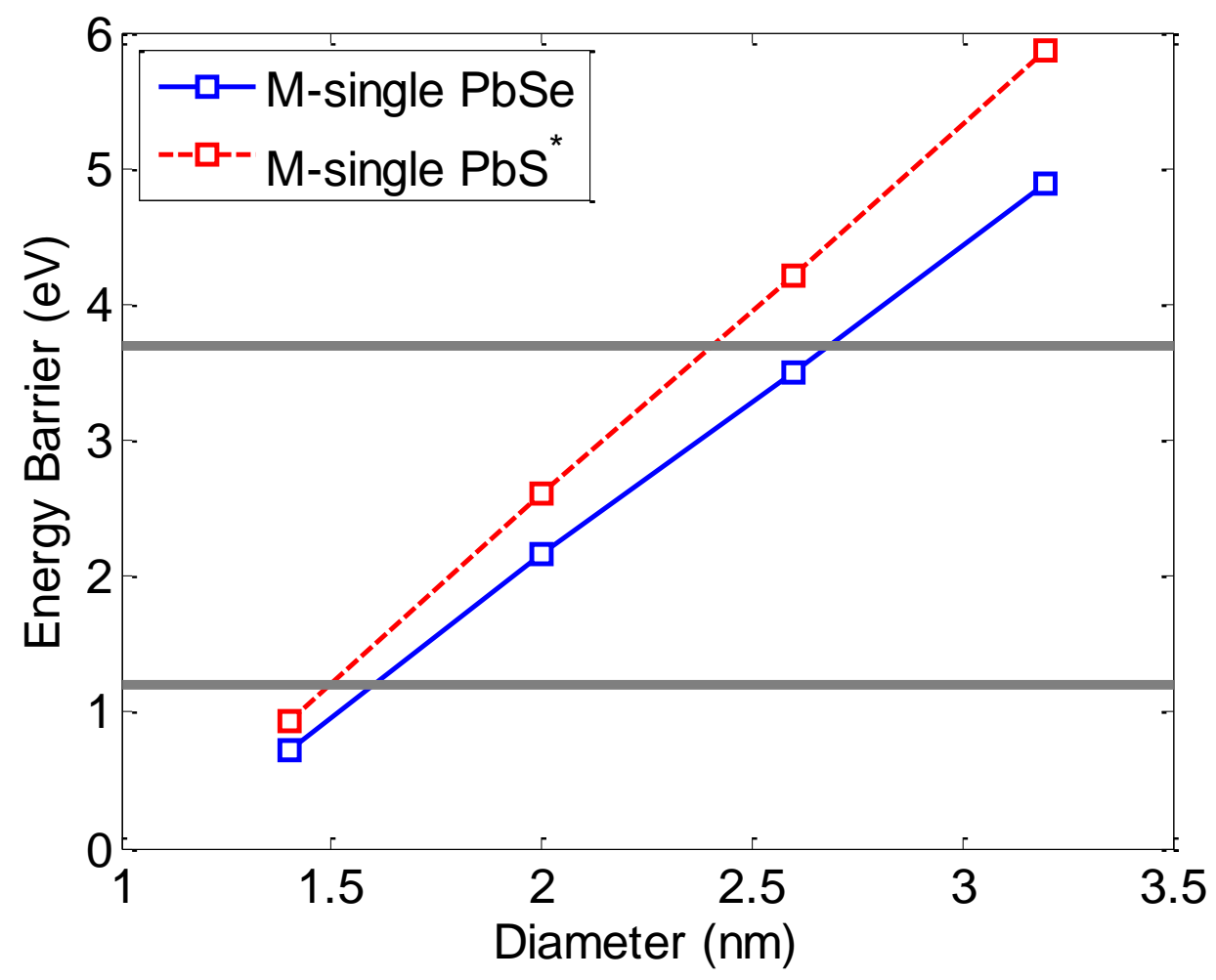

Figure 6. Energy barrier predicted by line tension model for a perfect dislocation for Eshelby dislocation escape from PbSe NWs as functions of NW diameter $D$ from the middle of NW. Two horizontal lines represent $1.2 \mathrm{eV}$ and $3.69 \mathrm{eV}$ which are threshold energy barrier at $300 \mathrm{~K}$ and $923 \mathrm{~K}$ (the growth temperature). "For PbS, energy barrier is zero for the mixed dislocation reported in experiments (Bierman et al., 2008) in the absence of the lattice resistance which is not considered in this study. Here, for a comparison, we present the energy barrier of $\mathrm{PbS}$ nanowire by assuming that it has only pure screw component.

We then apply the line tension model for a perfect dislocation to calculate the energy barrier for PbSe (Zhu et al., 2008) and PbS (Bierman et al., 2008) 〈100〉 NWs, in which Eshelby dislocations have been observed in experiments as non-dissociated perfect dislocations. The Burgers vectors of $\mathrm{PbSe}$ and $\mathrm{PbS}$ nanowires were reported to be $\langle 100\rangle \mathrm{a}$ and $\langle 110\rangle$ a, respectively, where a represent the lattice constant of each. In other words, pure screw dislocations were found in $\mathrm{PbSe}$ nanowires while mixed dislocations were found in $\mathrm{PbS}$ nanowires. For simplicity, we ignored the lattice resistance of the Halite crystals in line tension model, and escape from the end 
is not considered due to the lack of information on PbSe surface step energy. We find that the critical diameter at $1.2 \mathrm{eV}$ of PbSe NW turns out to be $1.6 \mathrm{~nm}$ at $300 \mathrm{~K}$, as depicted in Fig 6. At the growth temperature (923 K) (Zhu et al., 2008), the threshold energy barrier is $3.69 \mathrm{eV}$ and the critical diameter become $2.7 \mathrm{~nm}$, which is significantly smaller than the diameters observed in experiments, 50-200 nm (Zhu et al., 2008). Hence, NWs of these sizes are thermally stable and able to be grown via the screw-dislocation-driven growth. Unfortunately, the energy barrier of the mixed dislocation reported in PbS cannot be obtained by our model because the angle between the

Burgers vector and dislocation line exceed the critical value given by $\tan ^{-1}(\sqrt{1-v})$ (Hirth, 1958), making the mixed dislocation unstable for any diameter regardless of the magnitude of the surface step energy. Therefore, the Eshelby dislocation observed in $\mathrm{PbS}$ must be stabilized by lattice resistance, which is not yet accounted for in our model. For a comparison, we present the predicted energy barrier of $\mathrm{PbS}$ nanowire by assuming that it has only pure screw component in Table I and Fig 6. We find that such a dislocation is more stable in the PbS nanowire than that in the PbSe nanowire because of higher effective shear modulus.

\section{Summary}

In summary, we predict the stability of Eshelby dislocations in a range of FCC NW materials and diameters by combining atomistic and continuum models. The escape from the end of NW with an extended node is the preferred escape mode under all circumstances. This effect is more pronounced in materials with low stacking fault energy where the dislocation dissociation is wide. When the surface step is parallel to the dislocation's slip plane, the energy barrier is reduced dramatically, so that a much larger NW diameter is necessary to ensure stability at the experimental time scale. 


\section{Acknowledgements}

S. R. acknowledges the support of the Basic Science Research Program through the National Research Foundation of Korea (NRF) funded by the Ministry of Science, ICT \& Future Planning (2016R1C1B2011979). The work is partly supported by the U.S. Department of Energy, Office of Basic Energy Sciences, Division of Materials Science and Engineering under Award No. DESC0010412 (W.C.). We wish to thank William Kuykendall for discussions on the derivations of the line tension model. 


\section{Appendix A. Comparison between Atomistic Calculations and Experiments.}

We compare various materials properties that significantly affect the energetics of dislocation.

Because the atomistic potentials were fitted to the experimental values available for the authors, we find that our calculations match well with experimental results cited in the original atomistic potential papers (Ackland and Thetford, 1987; Ercolessi and Adams, 1994; Mishin et al., 2001; Rao et al., 1999) within small numerical errors (less than $1 \%$ ). To provide further detail, we compare the experimental data from an independent source (Lee et al., 2003), as depicted in Table A-1, which also shows a good agreement.

Table A-1. Comparison between atomistic calculations and experiments (Lee et al., 2003) for the various materials properties including lattice constant $\mathrm{a}_{0}$, cohesive energy $\mathrm{E}_{\mathrm{c}}$, vacancy formation energy $\mathrm{E}_{\mathrm{f}}$, three elastic constants, $\mathrm{C}_{11}, \mathrm{C}_{12}$, and $\mathrm{C}_{44}$, and stacking fault energy $\gamma_{\mathrm{SF}}$. Experimental results are given in the parentheses.

\begin{tabular}{|c|c|c|c|c|c|c|c|}
\hline & $\mathrm{a}_{0}(\AA)$ & $\mathrm{E}_{\mathrm{c}}(\mathrm{eV})$ & $\mathrm{E}_{\mathrm{f}}(\mathrm{eV})$ & $\mathrm{C}_{11}(\mathrm{GPa})$ & $\mathrm{C}_{12}(\mathrm{GPa})$ & $\mathrm{C}_{44}(\mathrm{GPa})$ & $\gamma_{\mathrm{SF}}\left(\mathrm{mJ} / \mathrm{m}^{2}\right)$ \\
\hline $\mathrm{Al}$ & 4.032 & 3.36 & 0.69 & 118 & 63 & 36.5 & 120.7 \\
& $(4.032)$ & $(3.36)$ & $(0.68)$ & $(114)$ & $(62)$ & $(32)$ & $(141)$ \\
\hline $\mathrm{Cu}$ & 3.615 & 3.54 & 1.27 & 169 & 122 & 75.3 & 44.6 \\
& $(3.615)$ & $(3.54)$ & $(1.03-1.30)$ & $(176)$ & $(125)$ & $(82)$ & $(42)$ \\
\hline $\mathrm{Ni}$ & 3.52 & 4.45 & 1.83 & 245 & 149 & 120 & 119.5 \\
& $(3.52)$ & $(4.45)$ & $(1.6)$ & $(261)$ & $(151)$ & $(132)$ & $(125)$ \\
\hline
\end{tabular}




\section{Appendix B. Single Dislocation Model from Hirth and Frank.}

In the original Hirth and Frank paper (Hirth and Frank, 1958), the energy of a straight mixed dislocation per unit length was given as

$$
E(y)=\frac{\mu b^{2}}{4 \pi}\left\{\cos ^{2} \phi\left[\ln \left(R^{2}-y^{2}\right)-\frac{\left(R^{2}-y^{2}\right)^{2}}{R^{4}}\right]+\frac{\sin ^{2} \phi}{1-v}\left[\ln \left(R^{2}-y^{2}\right)\right]\right\}
$$

where $\mathrm{y}$ is the distance from the NW axis. To be consistent with the energy expression for partial dislocations, Eq (2), used in this work, we do not include several constants in the original Hirth and Frank paper. The work of escape $\Delta \mathrm{W}$ can be written as

$$
\Delta W=\int_{-c / 2}^{c / 2}(E(y)-E(0)) d z-(s-c) \frac{\mu b^{2}}{4 \pi} \ln \frac{R}{r_{0}}
$$

where $\mathrm{s}$ is the arc length $2 \sqrt{h^{2}+(c / 2)^{2}}$, and the shape of dislocation is given as

$$
\mathrm{y}=\left\{\begin{array}{c}
h\left(1-\frac{2|z|}{c}\right), \quad|z| \leq \frac{1}{2} c \\
0, \quad|z|>\frac{1}{2} c
\end{array}\right.
$$

By evaluating the equation, we find the energy barrier being

$$
\begin{aligned}
\Delta W=\frac{\mu b^{2} c}{4 \pi}\left\{\left[\cos ^{2} \phi+\frac{\sin ^{2} \phi}{1-v}\right]\left[\ln \left(1-\frac{h^{2}}{R^{2}}\right)+\frac{R}{h} \ln \frac{1+h / R}{1-h / R}-2\right]\right. \\
\left.+\cos ^{2} \phi\left[\frac{2}{3}\left(\frac{h}{R}\right)^{2}-\frac{1}{5}\left(\frac{h}{R}\right)^{4}\right]+\left[\left(1+\frac{4 h^{2}}{c^{2}}\right)^{\frac{1}{2}}-1\right] \ln \frac{R}{r_{0}}\right\} .
\end{aligned}
$$

Now, we modify the original equation in Hirth and Frank by adding the orientation dependence in the line tenion, the last term in Eq. (A-2), as in the Eq. (1). 


$$
\begin{gathered}
\Delta W=\frac{\mu b^{2} c}{4 \pi}\left\{\left[\cos ^{2} \phi+\frac{\sin ^{2} \phi}{1-v}\right]\left[\ln \left(1-\frac{h^{2}}{R^{2}}\right)+\frac{R}{h} \ln \frac{1+h / R}{1-h / R}-2\right]+\cos ^{2} \phi\left[\frac{2}{3}\left(\frac{h}{R}\right)^{2}-\frac{1}{5}\left(\frac{h}{R}\right)^{4}\right]\right. \\
\left.+\left[\frac{f\left(\phi_{1}\right)+f\left(\phi_{2}\right)}{2}\left(1+\frac{4 h^{2}}{c^{2}}\right)^{\frac{1}{2}}-f(\phi)\right] \ln \frac{R}{r_{0}}\right\}
\end{gathered}
$$

$\phi_{1}$ and $\phi_{2}$ are the angle between the burgers vector and two inclined lines, as illustrated in Fig. 5 (a), and $f(\phi)=\cos ^{2} \phi+\frac{\sin ^{2} \phi}{1=v}$. For the screw dislocation $\left(\phi=0\right.$ and $\left.\phi_{1}=\phi_{2}\right)$, the energy barrier becomes

$$
\begin{gathered}
\Delta W=\frac{\mu b^{2} c}{4 \pi}\left\{\ln \left(1-\frac{h^{2}}{R^{2}}\right)+\frac{R}{h} \ln \frac{1+h / R}{1-h / R}-2+\frac{2}{3}\left(\frac{h}{R}\right)^{2}-\frac{1}{5}\left(\frac{h}{R}\right)^{4}\right. \\
\left.+\left[f\left(\phi_{1}\right)\left(1+\frac{4 h^{2}}{c^{2}}\right)^{\frac{1}{2}}-1\right] \ln \frac{R}{r_{0}}\right\} .
\end{gathered}
$$

The energy barrier $\mathrm{E}_{\mathrm{b}}$ is obtained at the value of $\mathrm{h}^{*}$ and $\mathrm{c}^{*}$ for which $\Delta W$ is simultaneously a maximum with respect to the first and a minimum with respect to the second. 


\section{References}

Ackland, G.J., Thetford, R., 1987. An improved N-body semi-empirical model for body-centered cubic transition metals. Philosophical magazine 56, 15-30.

Baimuratov, A.S., Rukhlenko, I.D., Gun'ko, Y.K., Baranov, A.V., Fedorov, A.V., 2015. Dislocation-induced chirality of semiconductor nanocrystals. Nano Lett 15, 1710-1715.

Berdichevsky, V.L., 2016. On a continuum theory of dislocation equilibrium. International Journal of Engineering Science 106, 10-28.

Bierman, M.J., Lau, Y.K.A., Kvit, A.V., Schmitt, A.L., Jin, S., 2008. Dislocation-driven nanowire growth and Eshelby twist. Science 320, 1060-1063.

Ercolessi, F., Adams, J.B., 1994. Interatomic Potentials from First-Principles Calculations: The ForceMatching Method. Europhys. Lett. 26, 583-588.

Eshelby, J.D., 1953. Screw Dislocations in Thin Rods. Journal of Applied Physics 24, 176-179.

Eshelby, J.D., 1979. Boundary Problems. Dislocations in Solids (Nabarro, F. R. N., ed.) 1, 167-221.

Eshelby, J.D., Stroh, A.N., 1951. Dislocations in Thin Plates. Philosophical magazine 42, 1401-1405.

Henkelman, G., Uberuaga, B.P., Jonsson, H., 2000. A climbing image nudged elastic band method for finding saddle points and minimum energy paths. J Chem Phys 113, 9901-9904.

Hirth, J.P., Frank, F. C., 1958. On the stability of dislocations in metal whiskers. Philosophical Magazine 3, 1110-1116.

Kang, K., Bulatov, V.V., Cai, W., 2012. Singular orientations and faceted motion of dislocations in bodycentered cubic crystals. Proceedings of the National Academy of Sciences of the United States of America 109, 15174-15178.

Kang, K., Yin, J., Cai, W., 2014. Stress Dependence of Cross Slip Energy Barrier for Face-Centered Cubic Metals. Journal of the Mechanics and Physics of Solids 62, 181-193.

Le, K.C., Piao, Y., 2016. Distribution of dislocations in twisted bars. International Journal of Plasticity 83, 110-125.

Lee, B.-J., Shim, J.-H., and Baskes, M.I., 2003. Semiempirical atomic potentials for the fcc metals $\mathrm{Cu}, \mathrm{Ag}$, $\mathrm{Au}, \mathrm{Ni}, \mathrm{Pd}, \mathrm{Pt}, \mathrm{Al}$, and $\mathrm{Pb}$ based on first and second nearest-neighbor modified embedded atom method. Physical Review B 68, 144112.

Leonardi, A., Ryu, S., Pugno, M.N., Scardi, P., 2015. Eshelby twist and correlation effects in diffraction from nanocrystals. JOURNAL OF APPLIED PHYSICS 117, 164304.

Madelung, O., Schulz, M., Weiss, H., 1983. Numerical Data and Functional Relationships in Science and Technology. Landolt-Bornstein, New Series (Springer, Berlin) 17.

Mai, L.Q., Tian, X.C., Xu, X., Chang, L., Xu, L., 2014. Nanowire Electrodes for Electrochemical Energy Storage Devices. Chem Rev 114, 11828-11862.

Meng, F., Jin, S., 2012. The Solution Growth of Copper Nanowires and Nanotubes is Driven by Screw Dislocations. Nano letters 12, 234-239.

Meng, F., Morin, S.A., Forticaux, A., Jin, S., 2013. Screw dislocation driven growth of nanomaterials. Acc Chem Res 46, 1616-1626.

Mishin, Y., Mehl, M.J., Papaconstantopoulos, D.A., Voter, A.F., Kress, J.D., 2001. Structural stability and lattice defects in copper: Ab initio, tight-binding, and embedded-atom calculations. Phys Rev B 63, 224106.

Palacios, T., 2012. APPLIED PHYSICS Nanowire electronics comes of age. Nature 481, 152-153.

Penner, R.M., 2012. Chemical Sensing with Nanowires. Annu Rev Anal Chem 5, 461-485.

Rao, S., Parthasarathy, T.A., Woodward, C., 1999. Atomistic Simulation of Cross-Slip Processes in Model FCC Structures. Philosophical magazine 79, 1167-1192.

Rasmussen, T., Jacobsen, K.W., Leffers, T., Pedersen, O.B., 1997. Simulations of the atomic structure, energetics, and cross slip of screw dislocations in copper. Phys Rev B 56, 2977-2990. 
Ren, Z., Gao, P.X., 2014. A review of helical nanostructures: growth theories, synthesis strategies and properties. Nanoscale 6, 9366-9400.

Roussel, J.M., Gailhanou, M., 2015. Stability of a Screw Dislocation in a 011 Copper Nanowire. Phys Rev Lett 115, 075503.

Ryu, I., Cai, W., Nix, W.D., Gao, H.J., 2016. Anisotropic Size-Dependent Plasticity in Face-Centered Cubic Micropillars Under Torsion. Jom-Us 68, 253-260.

Ryu, S., Kang, K., Cai, W., 2011. Entropic effect on the rate of dislocation nucleation. P Natl Acad Sci USA 108, 5174-5178.

Scattergood, R.O., Bacon, D.J., 1975. The Orowan mechanism in anisotropic crystals. Philosophical magazine 31, 179-198.

Scattergood, R.O., Bacon, D.J., 1982. The strenghening effect of voids. Acta Metallurgica 30, 1665-1677.

Weinberger, C.R., 2011. The structure and energetics of, and the plasticity caused by, Eshelby dislocations. International Journal of Plasticity 27, 1391-1408.

Weinberger, C.R., Cai, W., 2010a. Orientation-dependent plasticity in metal nanowires under torsion: twist boundary formation and Eshelby twist. Nano letters 10, 139-142.

Weinberger, C.R., Cai, W., 2010b. Plasticity of metal wires in torsion: Molecular dynamics and dislocation dynamics simulations. J Mech Phys Solids 58, 1011-1025.

Weinberger, C.R., Cai, W., 2012. Plasticity of metal nanowires. J Mater Chem 22, 3277-3292.

Xiong, X., Zou, C.L., Ren, X.F., Liu, A.P., Ye, Y.X., Sun, F.W., Guo, G.C., 2013. Silver nanowires for photonics applications. Laser Photonics Rev 7, 901-919.

Zhu, J., Peng, H.L., Marshall, A.F., Barnett, D.M., Nix, W.D., Cui, Y., 2008. Formation of chiral branched nanowires by the Eshelby Twist. Nat Nanotechnol 3, 477-481.

Zlotnikov, I., Werner, P., Fratzl, P., Zolotoyabko, E., 2015. Eshelby Twist as a Possible Source of Lattice Rotation in a Perfectly Ordered Protein/Silica Structure Grown by a Simple Organism. Small 11, 5636-5641. 